\title{
Effect of pressure on the pseudogap and charge density wave phases of the cuprate Nd-LSCO probed by thermopower measurements
}

\author{
A. Gourgout, ${ }^{1}$ A. Ataei, ${ }^{1}$ M.-E. Boulanger $\odot,{ }^{1}$ S. Badoux, ${ }^{1}$ S. Thériault $\odot,{ }^{1}$ D. Graf, ${ }^{2}$ J.-S. Zhou, ${ }^{3}$ S. Pyon ${ }^{\circledR},{ }^{4,5}$ T. Takayama, ${ }^{4,6}$ \\ H. Takagi, ${ }^{4,6,7,8}$ Nicolas Doiron-Leyraud $\odot,{ }^{1, *}$ and Louis Taillefer ${ }^{1,9, \dagger}$ \\ ${ }^{1}$ Institut Quantique, Département de physique and RQMP, Université de Sherbrooke, Sherbrooke, Québec J1K 2R1, Canada \\ ${ }^{2}$ National High Magnetic Field Laboratory, Florida State University, Tallahassee, Florida 32306, USA \\ ${ }^{3}$ Texas Materials Institute, University of Texas at Austin, Austin, Texas 78712, USA \\ ${ }^{4}$ Department of Advanced Materials Science, University of Tokyo, Kashiwa 277-8561, Japan \\ ${ }^{5}$ Department of Applied Physics, University of Tokyo, Tokyo 113-8656, Japan \\ ${ }^{6}$ Max Planck Institute for Solid State Research, Stuttgart 70569, Germany \\ ${ }^{7}$ Department of Physics, University of Tokyo, Tokyo 113-0033, Japan \\ ${ }^{8}$ Institute for Functional Matter and Quantum Technologies, University of Stuttgart, Stuttgart 70049, Germany \\ ${ }^{9}$ Canadian Institute for Advanced Research, Toronto, Ontario M5G 1M1, Canada
}

(Received 18 December 2020; revised 15 February 2021; accepted 10 March 2021; published 22 April 2021)

\begin{abstract}
We report thermopower measurements under hydrostatic pressure on the cuprate superconductor $\mathrm{La}_{1.6-x} \mathrm{Nd}_{0.4} \mathrm{Sr}_{x} \mathrm{CuO}_{4}$ (Nd-LSCO), at low temperature in the normal state accessed by suppressing superconductivity with a magnetic field up to $H=31 \mathrm{~T}$. Using an ac thermopower measurement technique suitable for high pressure and high field, we track the pressure evolution of the Seebeck coefficient $S$. At ambient pressure and low temperature, $S / T$ in Nd-LSCO was recently found to suddenly increase below the pseudogap critical doping $p^{\star}=0.23$, consistent with a drop in carrier density $n$ from $n=1+p$ above $p^{\star}$ to $n=p$ below. Under a pressure of $2.0 \mathrm{GPa}$, we observe that the large $S / T$ value just below $p^{\star}$ is suppressed. This confirms a previous pressure study based on electrical resistivity and Hall effect, which found that pressure lowers $p^{\star}$, thereby reinforcing the interpretation that this effect is driven by the pressure-induced shift of the van Hove point. It implies that the pseudogap only exists when the Fermi surface is hole-like, which puts strong constraints on theories of the pseudogap phase. We also report thermopower measurements on Nd-LSCO and $\mathrm{La}_{1.8-x} \mathrm{Eu}_{0.2} \mathrm{Sr}_{x} \mathrm{CuO}_{4}$ in the charge density wave phase near $p \sim 1 / 8$, which reveals a weakening of this phase under pressure.
\end{abstract}

DOI: 10.1103/PhysRevResearch.3.023066

\section{INTRODUCTION}

The pseudogap phase of cuprates is arguably one of their chief mysteries [1]. Understanding the pseudogap phase of cuprates has been hindered in part by the fact that it does not exhibit a clear and well-defined symmetrybreaking phase transition at its characteristic temperature $T^{\star}$. However, upon crossing the pseudogap end point at doping $p^{\star}$ (where $T^{\star}$ vanishes) at low temperatures, recent measurements have revealed a clear and abrupt change in carrier density $n$, going from $n=1+p$ above $p^{\star}$ to $n=$ $p$ below $p^{\star}$ [2]. These are based on high-field and lowtemperature measurements of the Hall effect on $\mathrm{YBa}_{2} \mathrm{Cu}_{3} \mathrm{O}_{y}$ (YBCO) [3] and $\mathrm{La}_{1.6-x} \mathrm{Nd}_{0.4} \mathrm{Sr}_{x} \mathrm{CuO}_{4}$ (Nd-LSCO) [4], resistivity on $\mathrm{La}_{2-x} \mathrm{Sr}_{x} \mathrm{CuO}_{4}$ (LSCO) [5] and Nd-LSCO [4],

\footnotetext{
*nicolas.doiron-leyraud@usherbrooke.ca

$\dagger$ louis.taillefer@usherbrooke.ca

Published by the American Physical Society under the terms of the Creative Commons Attribution 4.0 International license. Further distribution of this work must maintain attribution to the author(s) and the published article's title, journal citation, and DOI.
}

and thermal conductivity on Nd-LSCO [6]. Recently, the single-layer cuprates $\mathrm{Bi}_{2} \mathrm{Sr}_{2-x} \mathrm{La}_{x} \mathrm{CuO}_{6+\delta}(\mathrm{Bi2201})[7,8]$ and $\mathrm{Tl}_{2} \mathrm{Ba}_{2} \mathrm{CuO}_{6+\delta}(\mathrm{T} 12201)$ [8] were also shown to display such a drop in carrier density at $p^{\star}$ via Hall effect measurements, lending a sense of universality to these transport signatures of the pseudogap. Thermodynamic measurements have revealed a logarithmic divergence of the electronic specific heat at $p^{\star}$ [9], both as a function of doping and temperature, a classic signature of a quantum phase transition. Above $p^{\star}$, the resistivity of Nd-LSCO [10] and LSCO [11] exhibits a purely linear temperature dependence at low $T$ with a slope that reaches the Planckian limit [12], another signature of quantum criticality.

In the LSCO-based family of cuprates, an intriguing question is why do LSCO and Nd-LSCO have such a different $p^{\star}$, namely $p^{\star}=0.18-0.19$ for LSCO $[5,11,13]$, and $p^{\star}=0.23$ for Nd-LSCO [4]. This issue was examined via electrical resistivity and Hall effect measurements under hydrostatic pressure on Nd-LSCO [14], which found, using the drop in carrier density as a clear marker of $p^{\star}$, that $p^{\star}$ moves down with pressure $P$ at a rate of $d p^{\star} / d P \sim-0.01$ holes/GPa. This effect was shown to be driven by the pressure displacement of the van Hove point [14], where the Fermi surface changes from hole-like to electron-like, demonstrating that the pseudogap phase can only exist on a hole-like Fermi 
surface. In other words, the doping at which this Fermi surface change occurs, $p_{\mathrm{FS}}$, constrains the pseudogap phase, such that $p^{\star} \leqslant p_{\mathrm{FS}}$. In Nd-LSCO, $p_{\mathrm{FS}}=0.22 \pm 0.01[15]$ and $p^{\star}=0.23 \pm 0.01[4]$; in LSCO, $p_{\mathrm{FS}}=0.19 \pm 0.02[16,17]$ and $p^{\star}=0.18-0.19[5,11,13]$; in $\mathrm{Bi} 2201, p_{\mathrm{FS}}=0.41 \pm 0.02$ [18] and $p^{\star}=0.40 \pm 0.01[7,19]$. This explains why $p^{\star}$ has a different value in these different cuprates. The fact that $p^{\star} \leqslant p_{\text {FS }}$ places a strong constraint on candidate theories of the pseudogap phase, and it was found to be consistent with numerical solutions of the Hubbard model [20,21].

Recently, the thermopower was used as a probe of the carrier density across $p^{\star}$ in Nd-LSCO [22]. Unlike the Hall coefficient, $S$ does not depend sensitively on the curvature or shape of the Fermi surface. Unlike the conductivity (electrical or thermal), it does not depend sensitively on the level of impurity scattering. Within a simple model, in the $T=0$ limit and for a single band, the Seebeck coefficient of thermopower depends on two parameters only, the coefficient of electronic specific heat $\gamma=C_{e l} / T$ and the carrier density $n[23,24]$ ( $e$ is the electron charge):

$$
\frac{S}{T}=\frac{\gamma}{n e} .
$$

Fundamentally, $S / T$ is the specific heat per carrier. While seemingly oversimplistic, this expression was shown [23] to hold even in the presence of multiple bands and strong electronic correlations, as evidenced for a great variety of materials that includes common metals, oxides, heavy fermions, cuprates, and organic superconductors. In YBCO at $p=0.11$ for instance, there is excellent quantitative agreement between the normal-state $S / T$ measured in the $T \rightarrow 0$ limit $[25,26]$ and the estimate from Eq. (1) using the carrier density $n$ and the effective mass $m^{\star}$ obtained from quantum oscillations [27].

In the present paper, we report our pressure study of the thermopower of Nd-LSCO and $\mathrm{La}_{1.8-x} \mathrm{Eu}_{0.2} \mathrm{Sr}_{x} \mathrm{CuO}_{4}$ (Eu-LSCO), single-layer, tetragonal cuprate superconductors with a low critical temperature $T_{c}$ and field $H_{c 2}$, making them ideal candidates to study the field-induced normal-state Seebeck coefficient down to low temperatures. The phase diagram of Nd-LSCO is shown in Fig. 1(a), where the pseudogap temperature $T^{\star}$ extracted from resistivity measurements [4] is displayed and seen to be in agreement with angle-resolved photoemission spectroscopy (ARPES) measurements [15], showing that the transport signatures of the pseudogap in Nd-LSCO match those seen in spectroscopy [28]. In NdLSCO, recent thermopower measurements of the normal state found a sudden increase of $S / T$ below the pseudogap critical doping $p^{\star}=0.23$ [22]. This unambiguously confirms that the drop in $n$, from $n=1+p$ above $p^{\star}$ to $n=p$ below $p^{\star}$, first inferred from Hall effect, electrical resistivity, and thermal conductivity measurements is a genuine change in carrier density. At lower doping, in the vicinity of $p \sim 1 / 8$, charge density wave (CDW) order was previously shown to induce a negative $S / T$ at low temperature in YBCO [25], Eu-LSCO [25], $\mathrm{HgBa}_{2} \mathrm{CuO}_{4+\delta}(\mathrm{Hg} 1201)$ [29], and Nd-LSCO [22]. The aim of the present study is to use the clear signatures of the pseudogap and CDW phases in thermopower to study their evolution with pressure. To that effect, we recently developed an ac method which allows us to perform thermopower measurements under hydrostatic pressure and high magnetic field.

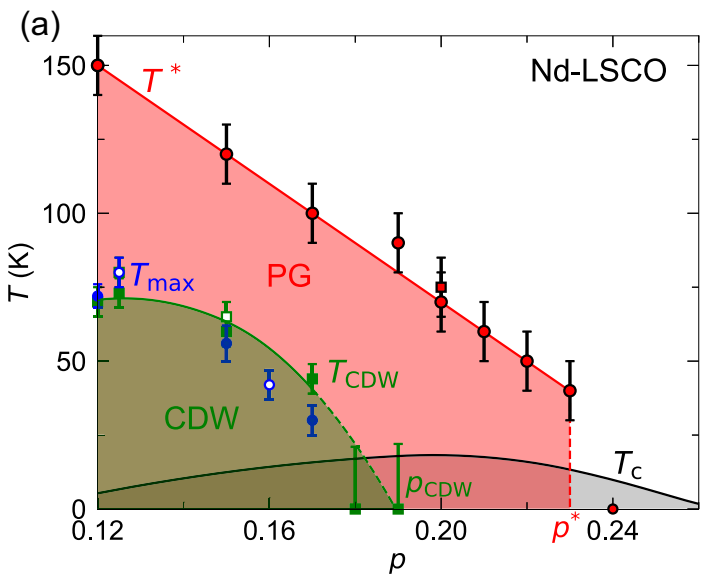

(b)

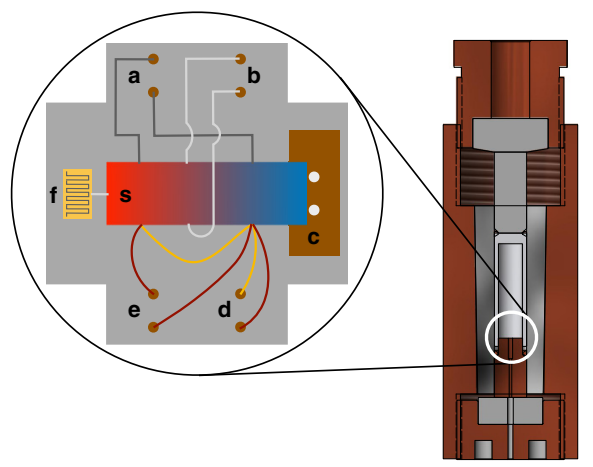

FIG. 1. (a) Temperature-doping phase diagram of Nd-LSCO showing the pseudogap temperature $T^{\star}$ extracted from resistivity (red dots $[4,22,28]$ ) and ARPES measurements (red square, from Ref. [15]), the CDW ordering temperature $T_{\mathrm{CDW}}$ as seen in x-ray diffraction measurements (green squares [30-32]), the temperature $T_{\max }$ of the maximum in $S / T$ vs $T$ (blue dots [22]), and a schematic of the zero-field superconducting transition temperature $T_{\mathrm{c}}$ (black line). Corresponding values for $T_{\max }$ [25] and $T_{\mathrm{CDW}}$ [33] in Eu-LSCO are shown as open symbols. The red and green full lines are guides to the eye. The red dashed line marks the end of the pseudogap phase, at the critical doping $p^{\star}=0.23 \pm 0.01$ [4]. The green dashed line is a linear extension of the full green line, extrapolating to $p=$ $p_{\mathrm{CDW}} \simeq 0.19$ at $T=0$. (b) Illustration of our experimental setup, showing the piston-cylinder pressure cell and a zoom on the top view of our thermopower mount at the tip of the electrical feedthrough. The mount shows the sample (s; red to blue gradient), differential and absolute type $\mathrm{E}$ thermocouples (e,d; red), phosphor-bronze wires for $V_{x}$ pick-up (a; grey), strain gauge sample heater (f; yellow), and copper heat sink (c; brown). The entire setup is mounted on a small $2 \mathrm{~mm} \times 2 \mathrm{~mm}$ G10 plate at the tip of our electrical feedthrough. Thermocouples are electrically insulated from the sample.

Our main finding is a clear suppression by pressure of the pseudogap signature in the thermopower of Nd-LSCO at $p=0.22$, just inside the pseudogap phase, while outside the pseudogap phase, at $p=0.24$, the thermopower shows a marginal change with pressure. This provides a clear and robust confirmation of the down shift of $p^{\star}$ with pressure first deduced from electrical transport measurements [14]. In Nd-LSCO and Eu-LSCO in the CDW phase, at $p \sim 1 / 8$, we observe a suppression of both the negative amplitude of $S / T$ and its sign-change temperature with pressure, which 
we interpret as a weakening of CDW order with pressure, as also seen in YBCO via transport [34], X-ray [35], and nuclear magnetic resonance (NMR) [36] measurements. We discuss the implications of our findings for the overall phase diagram of cuprates. Our results highlight the probing power of thermopower measurements under pressure.

\section{METHODS}

We measured the Seebeck coefficient using a low ac technique derived from the Ångström method [37,38] and adapted specifically for experiments in the pressure transmitting fluid encapsulated in a piston-cylinder pressure cell. Our experimental setup is displayed in Fig. 1(b). A thermal excitation was applied by sending an electrical current through a $120 \Omega$ strain gauge heater located on one side of the pressure chamber. The resulting longitudinal thermal gradient $\Delta T_{x}$ across the sample was measured using a type E differential thermocouple directly anchored to the sample. An absolute type E thermocouple measures the temperature on the cold side of the differential thermocouple, $T^{-}$, allowing a determination of the average temperature of the sample $T_{a v}=T^{-}+\Delta T_{x} / 2$. In all our measurements, $T_{a v}$ was found to be very close to $T_{0}$, the temperature of our Cernox sensor located just outside the pressure cell, meaning that the heat current generated a small $\Delta T_{x}$ without much elevation of the temperature inside the pressure cell. Note that type-E thermocouples have a weak field dependence in the field and temperature range of our measurements. The Seebeck voltage $\Delta V_{x}$ was measured with phosphor-bronze wires using the same contacts as $\Delta T_{x}$, which eliminates uncertainties associated with the geometric factor. The Seebeck coefficient is then given by $S=-\Delta V_{x} / \Delta T_{x}$. For the heat current, we use a low ac square-wave electrical current oscillating between zero and twice the wave amplitude, at a typical frequency of $400 \mathrm{mHz}$. The thermocouples and Seebeck voltages are amplified using preamplifiers based on EM Electronics A10 chips and picked up using SR830 lock-in amplifiers at the thermal excitation frequency. Our ac method provides two major advantages over the usual steady-state dc technique: (1) a major boost in measuring speed, which allows recording $S(T)$ continuously from 2 to $300 \mathrm{~K}$ within a few hours and, (2) a greater stability against noise and perturbations that typically plague dc measurements. We carefully benchmarked our approach against the dc method and found no significant difference.

Pressure was applied on our samples using a miniature nonmagnetic piston-cylinder cell. The pressure medium is Daphne oil 7474, which remains liquid at all pressures measured here at $300 \mathrm{~K}$, ensuring a high degree of hydrostaticity. The internal pressure is measured both at room temperature and at $4.2 \mathrm{~K}$, using either the fluorescence of a small ruby chip or a $\mathrm{Sn}$ manometer. The values quoted throughout are the low temperature pressures. The error bar on all the pressure values is $\pm 0.05 \mathrm{GPa}$, which comes from the uncertainty in measuring the position of the fluorescence peaks. For each measurement, the cell was cooled slowly $(<1 \mathrm{~K} / \mathrm{min})$ to ensure a homogeneous freezing of the pressure medium.

Large single crystals of Nd-LSCO were grown at Texas Materials Institute by a traveling float-zone technique in an image furnace, with nominal $\mathrm{Sr}$ concentrations $x=0.12$,
0.22 , and 0.24 . Two of these samples (0.22 and 0.24$)$ were previously measured by electrical resistivity and Hall effect [4], and all three were studied by thermal conductivity [9] (sample details can be found in these references). Our crystal of EuLSCO with $x=0.125$ was grown in Tokyo. Thermopower measurements on a closely related sample were previously reported in Ref. [25], further sample details can be found there. The hole concentration $p$ of each sample is given by $p=x$.

Samples were cut into small rectangular platelets of typical dimensions $1 \mathrm{~mm} \times 0.5 \mathrm{~mm} \times 0.2 \mathrm{~mm}$, with the shortest dimension along the $c$ axis. Contacts were made with $\mathrm{H} 20 \mathrm{E}$ silver epoxy diffused by annealing at high temperature in flowing oxygen. Thermopower measurements under pressure in magnetic fields up to $18 \mathrm{~T}$ were performed at Sherbrooke, and up to $31 \mathrm{~T}$ at the NHMFL in Tallahassee. We measured the thermopower in the $a b$-plane, along the longest sample dimension. The magnetic field was applied along the $c$ axis and the Seebeck voltage signal was symmetrized with respect to field inversion in order to remove contaminations from the Nernst effect.

\section{RESULTS AND DISCUSSION}

\section{A. Pseudogap phase at ambient pressure}

In Fig. 2, we show isotherms of $S / T$ as a function of magnetic field $H$ up to $31 \mathrm{~T}$ for Nd-LSCO at $p=0.22$ and 0.24 at 0.1 and $2.0 \mathrm{GPa}$. With increasing field, $S / T$ is null in the superconducting state at low field, then rises quickly upon crossing the vortex solid melting field $H_{\mathrm{vs}}$, and finally reaches the normal-state value above the upper critical field $H_{c 2}$. At both dopings, we observe a rise of the normal-state $S / T$ with decreasing temperature. These findings are in agreement with our previous report of the zero-pressure thermopower in Nd-LSCO [22]. In particular, as shown in Fig. 3(a), our data inside a pressure cell at the lowest pressure of $0.1 \mathrm{GPa}$ are in excellent quantitative agreement with ambient pressure data on the same sample, free-standing and measured using a standard steady-state dc method [22], which demonstrates the reliability of our pressure setup. This holds for both $p=$ 0.22 and 0.24 . As in Ref. [22], these curves for $S / T$ versus $T$ as $H \rightarrow 0$ are constructed from the isotherms shown in Fig. 2, using the linear fits to the normal-state data above $H_{c 2}$, back-extrapolated to $H=0$ in order to capture the intrinsic normal-state $S / T$ free from the sample-dependent negative field dependence.

At $P=0.1 \mathrm{GPa}$ [Figs. 2(a) and 2(b)], the curves of $S / T(H \rightarrow 0)$ yield, at $5 \mathrm{~K}, S / T=0.45$ and $2.1 \mu \mathrm{V} / \mathrm{K}^{2}$ at $p=0.24$ and 0.22 , respectively, roughly a 5-fold increase. As a function of doping, this increase in $S / T(H \rightarrow 0)$ suddenly occurs at $p^{\star}$, as shown in Fig. 4(a) and reported in Ref. [22], and constitutes a clear thermopower signature of the pseudogap phase. It confirms and reinforces the pseudogap signatures in the electrical resistivity $\rho$ and Hall coefficient $R_{\mathrm{H}}$ [4], as shown in the top panels of Fig. 3 where the parallel lowtemperature upturns are seen in $S / T, \rho$, and $R_{\mathrm{H}}$ in Nd-LSCO $p=0.22$. As a result, in the $T \rightarrow 0$ limit the normal-state values of $\rho$ and $S / T$ display a comparable increase between $p=0.24$ and 0.22 , by a factor of about 5 (Fig. 4). Since, in a simple model, $S / T \propto m^{\star} / n, \rho \propto m^{\star} / \tau n$, and $R_{\mathrm{H}} \propto 1 / n$, 

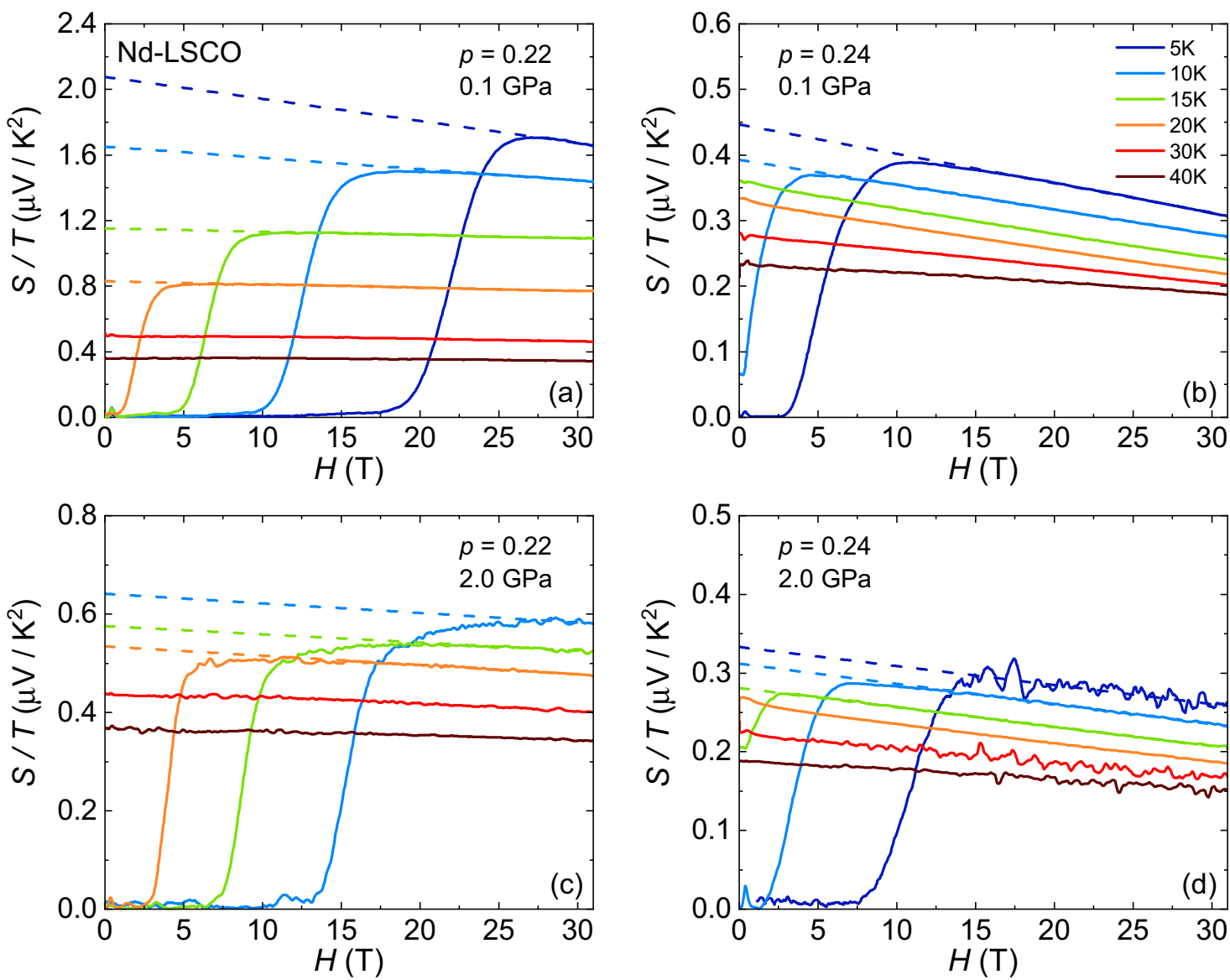

FIG. 2. Isotherms of the Seebeck coefficient expressed as $S / T$ versus magnetic field $H$ in Nd-LSCO at $p=0.22$ [(a), (c)] and $p=0.24$ $[(\mathrm{b}),(\mathrm{d})]$, at temperatures and pressures as indicated. At near ambient pressure $(0.1 \mathrm{GPa})[(\mathrm{a}),(\mathrm{b})], S / T$ changes by a factor of about 5 when going from $p=0.22<p^{\star}$ to $p=0.24>p^{\star}$, indicative of the sudden drop in carrier density at the onset of the pseudogap phase [22]. Under $2.0 \mathrm{GPa}, S / T$ at $p=0.22$ is heavily suppressed, signaling a lowering of $p^{\star}$ with pressure. Dashed lines are linear fits to the isotherms at high field, whose back-extrapolation to $H=0$ yields the values of $S / T$ at $H \rightarrow 0$ (see text).

where $1 / \tau$ is the scattering rate, this strongly suggests that it is a drop in carrier density that causes the jump in the three quantities across $p^{\star}$. Note that the $50 \%$ decrease in the electronic specific heat between $p=0.24$ and 0.22 [9] reinforces our conclusion that the carrier density must decrease by a large factor when $p$ is reduced below $p^{\star}$. As for YBCO [3] and Bi2201 [7], these transport signatures in Nd-LSCO are all indicative of a drop in carrier density at $p^{\star}$, with $n$ going from $n=1+p$ above $p^{\star}$ to $n=p$ below.

Let us stress two key aspects here. First, in Nd-LSCO the van Hove singularity in the specific heat, which coincides with $p^{\star}$, is cut off because of substantial $c$-axis dispersion and disorder. This was shown through calculations of the effect of the van Hove singularity on the specific heat in Nd-LSCO [9] and confirmed through ARPES measurements [39]. As a result, the pseudogap phase is the chief driving force for the change in carrier density at $p^{\star}$. Second, magnetoresistance [4] and angle-resolved magnetoresistance [40] have shown that the scattering rate does not change substantially across $p^{\star}$, which reinforces the conclusion that the change at $p^{\star}$ is in the carrier density.

Going just outside the pseudogap phase, at $p=0.24$, NdLSCO instead displays signatures of quantum criticality, as first noted by the linear- $T$ resistivity which extends down to the lowest measured temperature [10] [Fig. 3(b)] and whose slope was recently shown to obey the Planckian limit [12], seen in several other quantum critical metals [41]. Recent specific-heat measurements on Nd-LSCO revealed $C_{e l} / T \propto$ $\log (1 / T)$ at $p=0.24$ [9], another signature of quantum criticality [42]. A logarithmic divergence is also seen in the Seebeck coefficient, with $S / T \propto \log (1 / T)$ at $p=0.24$ in both Nd-LSCO [43] and Eu-LSCO [25].

\section{B. Pseudogap phase at 2.0 GPa}

Our main result is displayed in Fig. 3(d): The lowtemperature upturn in $S / T$ at $p=0.22$ is fully suppressed under a pressure of $2.0 \mathrm{GPa}$, with $S / T$ now increasing only very slowly with decreasing temperature. Quantitatively, $S / T$ at $p=0.22$ at $5 \mathrm{~K}$ and $31 \mathrm{~T}$ goes from $1.8 \mu \mathrm{V} / \mathrm{K}^{2}$ in $0.1 \mathrm{GPa}$ to $0.7 \mu \mathrm{V} / \mathrm{K}^{2}$ in $2.0 \mathrm{GPa}$. As a result of this suppression, $S / T$ at $p=0.22$ and $2.0 \mathrm{GPa}$ displays essentially the same temperature evolution as that for $p=0.24$ at $0.1 \mathrm{GPa}$, except for a rigid shift. Note that we show continuous $T$ sweeps of $S / T$ in $H=31 \mathrm{~T}$ taken using our ac method, something not possible with a standard dc technique.

This pressure suppression of $S / T$ at $p=0.22$ mirrors the suppression first observed in $\rho$ and $R_{\mathrm{H}}$ [14] and displayed for 

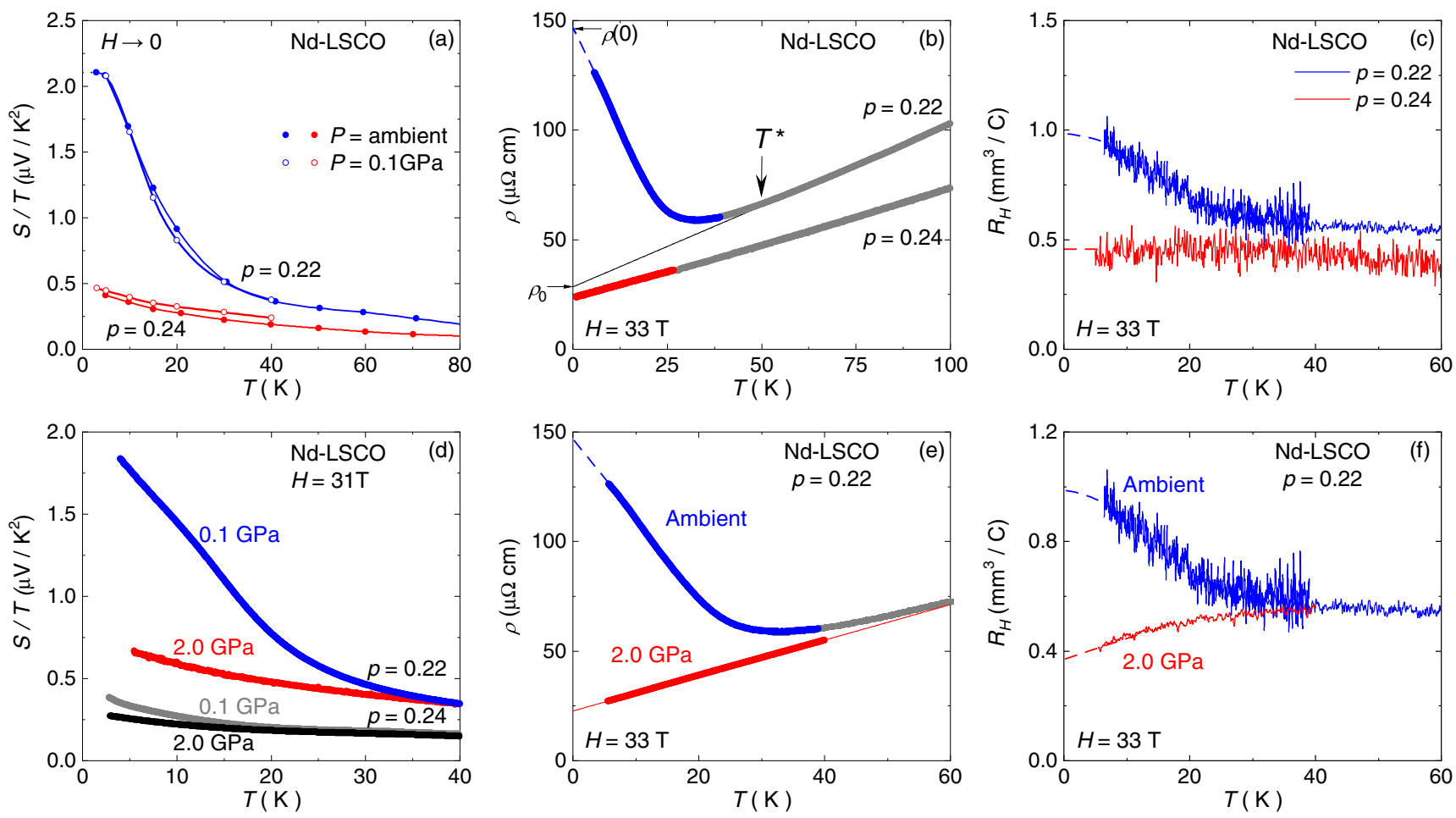

FIG. 3. Pressure effects on the transport properties of Nd-LSCO near $p^{\star}$. Top panels (ambient pressure): (a) Seebeck coefficient $S / T$, (b) electrical resistivity $\rho$, and (c) Hall coefficient $R_{\mathrm{H}}$ in Nd-LSCO at $p=0.22$ (blue) and 0.24 (red), in the field-induced normal state. Data for $S / T$ are from the present study (0.1 GPa; open circles) and from Ref. [22] (ambient; dots), in the $H \rightarrow 0$ limit obtained via back-extrapolations as shown in Fig. 2. Data for $\rho$ and $R_{\mathrm{H}}$ are reproduced from Ref. [4], and are respectively in zero-field (grey) and $H=16 \mathrm{~T}$ at high temperature, and $H=33 \mathrm{~T}$ at low temperature. In panel (b), we label the value of $\rho$ at $p=0.22$ and $H=33 \mathrm{~T}$, extrapolated to $T \rightarrow 0$, as $\rho(0)$, and the value obtained from a linear extrapolation of the high temperature $T$-linear regime as $\rho_{0}$. Bottom panels (2.0 GPa): data on Nd-LSCO at $p=0.22$ in $2.0 \mathrm{GPa}$, showing a clear suppression of the low-temperature upturns in $S / T$ (d), $\rho$ (e), and $R_{\mathrm{H}}$ (f). In panel (d) we also show $S / T$ for Nd-LSCO $p=0.24$ in 0.1 and $2.0 \mathrm{GPa}$. Data for $S / T$ are from the present study in $H=31 \mathrm{~T}$. Data for $\rho$ and $R_{\mathrm{H}}$ are reproduced from Ref. [14], in fields as indicated.

the same doping in Figs. 3(e) and 3(f): in $2.0 \mathrm{GPa}, \rho$ goes from showing a huge low-temperature upturn at ambient pressure to displaying a pure linear- $T$ behavior, and $R_{\mathrm{H}}$ looses its upturn and becomes flat. Having now the Seebeck coefficient showing the same suppression with pressure demonstrates that the effect is not a peculiarity of $\rho$ or $R_{\mathrm{H}}$, but is the reflection of a genuine suppression of the drop in carrier density accompanying the pseudogap phase, with all three coefficients at $p=0.22$ and $2.0 \mathrm{GPa}$ displaying the same behavior as at $p=0.24$, where there is no pseudogap. In contrast, we note that 2.0 GPa has a marginal effect on our $p=0.24$ sample, slightly flattening $S / T$ at low temperature [Fig. 3(d)]. Another effect of pressure is a strengthening of superconductivity at $p=0.22$, as indicated by the rise of $T_{c}$ and $H_{c 2}$ (Fig. 2).

We observe that pressure impacts only the low-temperature behavior, with the curves of $S / T, \rho$, and $R_{\mathrm{H}}$ in $2.0 \mathrm{GPa}$ all merging with the ambient pressure (or $0.1 \mathrm{GPa}$ ) curves at some temperature above $40 \mathrm{~K}$ or so [Figs. 3(d)-3(f)]. Pressure also does not change the actual doping in Nd-LSCO, which is determined by the $\mathrm{Sr}$ content. In Fig. 4(a), we show our data for $S / T$ at $5 \mathrm{~K}$ and $H \rightarrow 0$ at $2.0 \mathrm{GPa}$ and observe that the point at $p=0.22$ naturally extrapolates the line of $S / T$ vs $p$ above $p^{\star}$, showing that $p^{\star}$ itself has moved to lower dopings in $2.0 \mathrm{GPa}$. Based on our resistivity data under pres- sure [14], shown in Fig. 4(b), we see that $p^{\star}$, as signaled by the sudden jump in $\rho(0) / \rho_{0}$, has in fact moved from 0.23 to 0.21 in $2.0 \mathrm{GPa}$. This implies a rate of suppression of $d p^{\star} / d P \simeq-0.01$ holes/GPa, consistent with our Seebeck data at $p=0.22$. We expect that $S / T$ at $p=0.21$ should also display a near full suppression under 2.0 GPa. As discussed in Ref. [14], we stress that pressure has no effect on the pseudogap temperature $T^{\star}$ itself, so the suppression of $p^{\star}$ is not accompanied by an overall collapse of $T^{\star}$.

Consistent with the fact that $p^{\star}$ moves down with pressure, we observe that $S / T$ at $p=0.22$ and 0.24 , which are both outside the pseudogap phase in $2.0 \mathrm{GPa}$, exhibit the same slow growth with decreasing temperature but with a roughly 2-fold difference in size [Fig. 3(d)]. This is the same factor by which the inelastic part of the resistivity, which is perfectly $T$-linear, changes between $p=0.22$ and 0.24 [Figs. 3(b) and 3(e)]. Given that $S / T \propto m^{\star} / n, \rho \propto$ $m^{\star} / \tau n$, and that $n$ varies little over this doping range (for $\left.p>p^{\star}\right)$, we infer that the factor of 2 must come from an increase of $m^{\star}$ upon approaching $p^{\star}$ from above, as argued in Ref. [12]. Such an increase of $m^{\star}$ is consistent with specificheat data on Nd-LSCO [9] that show an increase of $C_{e l} / T$ as $p$ is lowered towards $p^{\star}$ from above. So, with $p^{*}$ pushed to below $p=0.22$ in $2.0 \mathrm{GPa}$, quantitative values of $S / T$ 

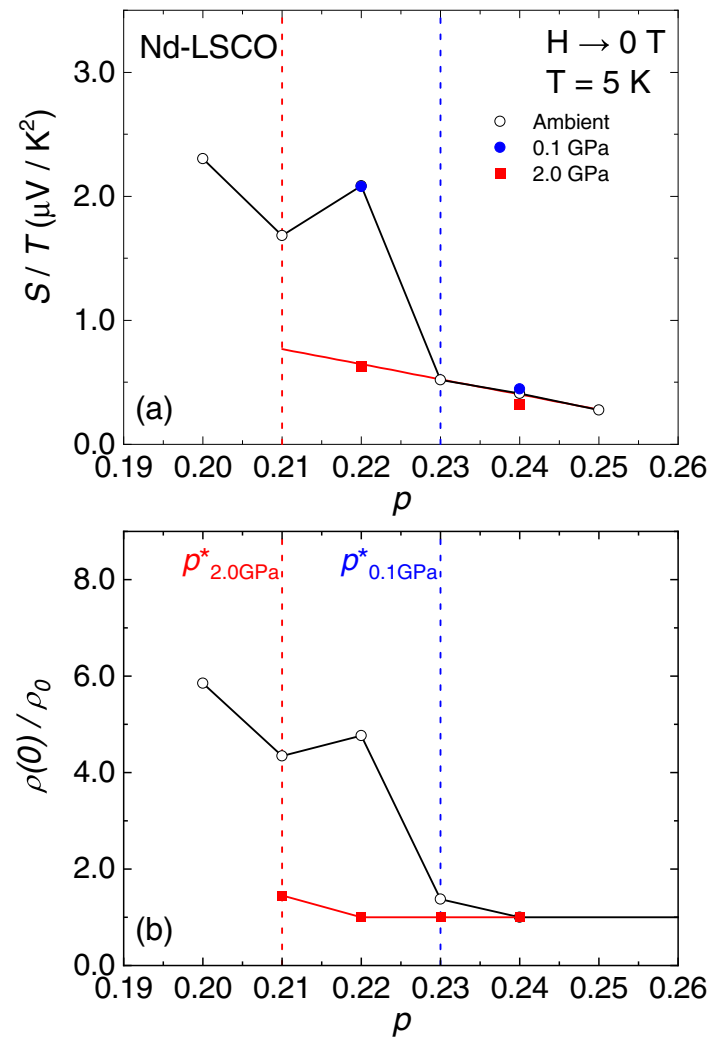

FIG. 4. (a) Seebeck coefficient $S / T$ versus doping $p$ for NdLSCO, measured at $T=5 \mathrm{~K}$ in the $H \rightarrow 0$ limit. Data at ambient pressure (open circles) are reproduced from [22]. Data at $P=$ $0.1 \mathrm{GPa}$ (blue dots) and $2.0 \mathrm{GPa}$ (red dots) are from the present study. The vertical blue dashed line indicates $p^{\star}$ at ambient pressure, which coincides with the onset of the rise of $S / T$ caused by the drop in carrier density [4,22]. Upon application of $2.0 \mathrm{GPa}$ this rise in $S / T$ is fully suppressed at $p=0.22$, and $S / T$ now follows the doping evolution extrapolated from outside the pseudogap phase (red line). (b) Ratio $\rho(0) / \rho_{0}$ [see Fig. 3(b)] as a function of doping, at ambient pressure (open circles [4]) and 2.0 GPa (red dots [14]), which puts $p^{\star}$ in $2.0 \mathrm{GPa} p^{\star} \simeq 0.21$ [14], as shown by the vertical red dashed line. The present data for $S / T$ are consistent with such a pressure-induced suppression of $p^{\star}$.

and $\rho$ are consistent with the doping evolution expected at $p>p^{\star}$.

The fact that the shift of $p^{\star}$ under pressure is now seen in thermopower confirms and reinforces the conclusion laid out in Ref. [14], namely that the shift of $p_{\mathrm{FS}}$ with pressure is driving a corresponding shift in $p^{\star}$, such that $p^{\star} \leqslant p_{\mathrm{FS}}$ continues to be obeyed. Above $p_{\mathrm{FS}}$, the Fermi surface is electron-like and the Hall coefficient $R_{\mathrm{H}}$ is seen to linearly decrease with doping $[44,45]$, reaching negative values well above $p_{\mathrm{FS}}$. Consequently, a relative change of $R_{\mathrm{H}}$ with pressure at constant doping indicates that $p_{\mathrm{FS}}$ itself is decreasing with pressure, as observed [14] and expected from band-structure calculations [14]. The shift of $p_{\mathrm{FS}}$ and $p^{\star}$ in $2.0 \mathrm{GPa}$ were found to match, both moving by about 0.02 holes per planar $\mathrm{Cu}$ atoms [14]. That $p^{\star} \leqslant p_{\mathrm{FS}}$ must be obeyed explains why LSCO, Nd/EuLSCO, and Bi2201 all have different $p^{\star}$ values, and shows that the pseudogap can only exist on a hole-like Fermi surface,
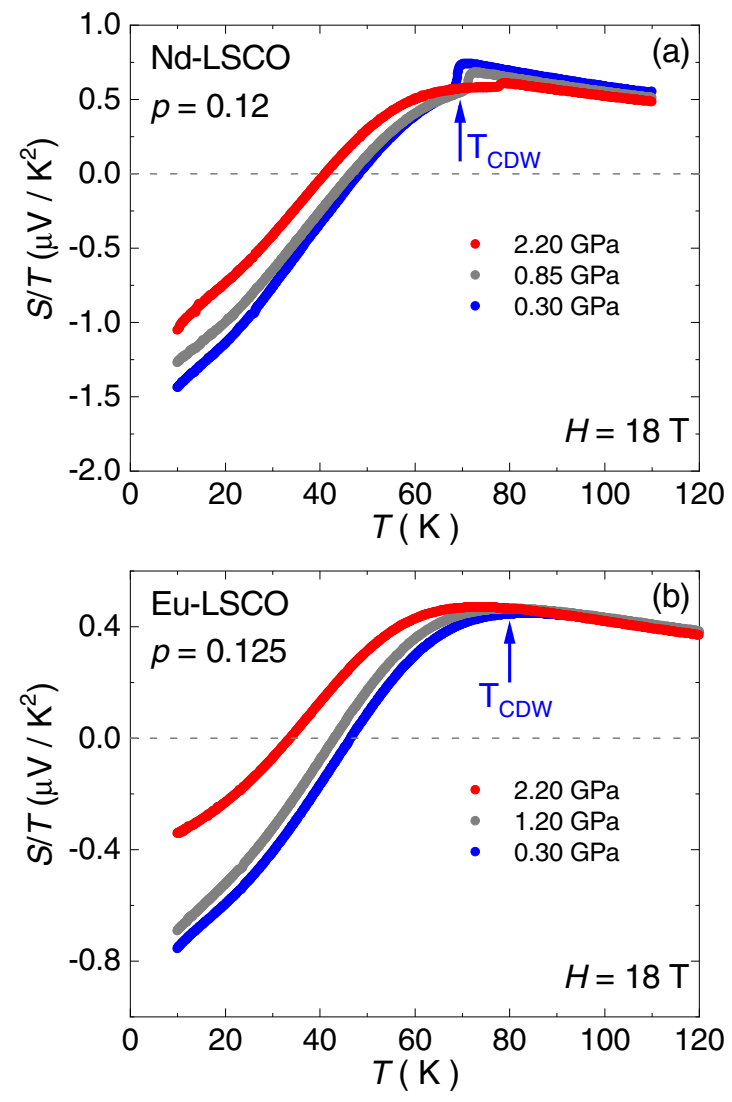

FIG. 5. Seebeck coefficient $S / T$ vs $T$ in the CDW phase, in (a) Nd-LSCO at $p=0.12$ and (b) Eu-LSCO at $p=0.125$, in the field-induced normal state at $H=18 \mathrm{~T}$ and at pressures as indicated. In Nd-LSCO $p=0.12$, x-ray diffraction measurements [30,31] detect the onset of CDW order at $T_{\mathrm{CDW}}=70 \mathrm{~K}$, which coincides with the maximum in $S / T$ at $T_{\max } \simeq 70 \mathrm{~K}$. The step in the data is caused by the LTO-LTT structural transition at $T_{\mathrm{LTT}} \simeq 70 \mathrm{~K}$. In Eu-LSCO $p=0.125$, the $\mathrm{CDW}$ order occurs at $T_{\mathrm{CDW}} \simeq 80 \mathrm{~K}$ [33] while the structural transition is at $T_{\mathrm{LTT}} \simeq 130 \mathrm{~K}$ [46].

which imposes a stringent constraint on theories of the pseudogap phase. This was also found in numerical calculations of the Hubbard model [20,21].

\section{Pressure effect on charge density wave phase}

We now turn to dopings well below $p^{\star}$ and at the center of the CDW phase, namely $p=0.12$ in Nd-LSCO and $p=$ 0.125 in Eu-LSCO. As displayed in Fig. 1(a), x-ray diffraction measurements $[30,31]$ detect the onset of $\mathrm{CDW}$ order in Nd-LSCO $p=0.12$ at a temperature $T_{\mathrm{CDW}}=70 \mathrm{~K}$. Our data for $S / T$ vs $T$ for this sample show a clear departure from the data at $p=0.22$, with $S / T$ at near ambient pressure ( $0.3 \mathrm{GPa}$ ) going through a broad maximum at $T_{\max } \simeq 70 \mathrm{~K}$ before falling to negative values at low temperatures [Fig. 5(a)]. Eu-LSCO at $p=0.125$ exhibits a similar behavior for $S / T$ [Fig. 5(b)], while x-ray measurements find a comparable $T_{\mathrm{CDW}}$ close to $80 \mathrm{~K}$ [33]. A negative $S / T$ is typical of cuprates near $p \simeq 1 / 8$, as observed in LSCO [47], LBCO [48], Nd-LSCO [49,50], Eu-LSCO [50,51], YBCO [25,51], and Hg1201 [29]. These studies showed that a negative Seebeck coefficient is 
a consequence of the Fermi surface reconstruction caused by the CDW order. In both Nd-LSCO and Eu-LSCO, this is supported by the fact that $T_{\max }$ coincides with $T_{\mathrm{CDW}}$ [Fig. 1(a)] [22]. This recently allowed us to establish the doping range of the CDW phase in Nd-LSCO, present up to about $p=$ $p_{\mathrm{CDW}} \simeq 0.19$ [22], in agreement with a recent $\mathrm{x}$-ray study of Nd-LSCO [32] [see Fig. 1(a)]. Note that in Nd-LSCO $p=$ 0.12 , the structural transition from the low-temperature orthorombic (LTO) to low-temperature tetragonal (LTT) phase also coincides with $T_{\mathrm{CDW}}$ and $T_{\mathrm{max}}$, causing the sharp step in our $S / T$ data [Fig. 5(a)]. In Eu-LSCO, the LTO-LTT transition occurs at a much higher temperature, with $T_{\mathrm{LTT}} \simeq 130 \mathrm{~K}$ [46] at $p=0.125$, and yet it displays the same $S / T$ curve as Nd-LSCO, showing that it is the CDW, and not the LTO-LTT transition, which is causing the negative $S / T$ [25].

In both Nd-LSCO and Eu-LSCO, we observe two clear effects under increasing pressure: (1) at low temperatures, the amplitude of the negative $S / T$ is suppressed and (2) the temperature $T_{\max }$ shifts down. In Eu-LSCO, the suppression of $S / T$ is significant, going at $10 \mathrm{~K}$ from about $-0.8 \mu \mathrm{V} / \mathrm{K}^{2}$ at $0.3 \mathrm{GPa}$ to $-0.35 \mu \mathrm{V} / \mathrm{K}^{2}$ at $2.0 \mathrm{GPa}$, a $50 \%$ change. $T_{\max }$ goes from about $80 \mathrm{~K}$ at $0.3 \mathrm{GPa}$ to $70 \mathrm{~K}$ at $2.0 \mathrm{GPa}$. As a result, the sign-change temperature is also clearly suppressed by pressure, moving from $47 \mathrm{~K}$ at $0.3 \mathrm{GPa}$ to $32 \mathrm{~K}$ at $2.0 \mathrm{GPa}$. Similar effects are seen on Nd-LSCO, albeit smaller in amplitude. Given that the negative $S / T$ is a clear marker of the CDW phase, its suppression is clear indication that the CDW phase is weakened by pressure. Note that the high-temperature $S / T$ above $T_{\max }$ is only weakly affected by the pressure, showing that pressure principally impacts the CDW phase.

Note also that superconductivity (SC) is boosted with pressure, as evidenced by the increase in $T_{c}$ we observe in both $\mathrm{Nd}-\mathrm{LSCO}$ and Eu-LSCO (at $p \simeq 0.12, T_{\mathrm{c}}$ rises from $7 \mathrm{~K}$ at $0.3 \mathrm{GPa}$ to $15 \mathrm{~K}$ at $2.2 \mathrm{GPa}$ ), suggesting a competition between SC and CDW. This pressure tuning of the competition between SC and CDW was previously inferred in YBCO based on resistivity and Hall effect measurements under hydrostatic pressure [34]. In YBCO, the suppression of CDW modulations with pressure was directly observed by $\mathrm{x}$-ray diffraction [35] and NMR [36] measurements. Interestingly, in the case of Nd-LSCO $p=0.12$ we observe a clear upward shift of $T_{\text {LTT }}$ with pressure, in agreement with a previous X-ray study at the same doping [52].

\section{SUMMARY}

We have used the Seebeck effect to examine the pressure dependence of the pseudogap critical point $p^{\star}$ in the cuprate superconductor Nd-LSCO, and of the CDW phase in both Nd-LSCO and Eu-LSCO. We observe that the large Seebeck coefficient inside the pseudogap phase at $p=0.22$, that results from the low carrier density below $p^{\star}$, is fully suppressed under a pressure $P=2.0 \mathrm{GPa}$. This confirms and reinforces our previous observation, inferred from resistivity and Hall effect measurements [14], that $p^{\star}$ in Nd-LSCO shifts down with pressure and is fundamentally driven by a shift in pressure of the van Hove point where the Fermi surface changes from hole-like to electron-like. This strengthens the notion that the pseudogap phase only exists on a hole-like Fermi surface, which implies important theoretical constraints. At lower doping, at $p \simeq 1 / 8$ where the CDW phase is strongest, we observe in both $\mathrm{Nd}-\mathrm{LSCO}$ and Eu-LSCO a reduction in magnitude of the negative Seebeck coefficient with increasing pressure, which we attribute to a weakening of the CDW order with pressure. This confirms in Nd-LSCO and Eu-LSCO the phase competition between CDW order and superconductivity seen in other cuprates.

\section{ACKNOWLEDGMENTS}

We thank K. Behnia, A. Georges, J. Mravlje, A.-M. Tremblay, and S. Verret for fruitful discussions. We thank S. Fortier and M. Dion for their assistance with the experiments. L.T. acknowledges support from the Canadian Institute for Advanced Research (CIFAR) as a CIFAR Fellow and funding from the Institut Quantique, the Natural Sciences and Engineering Research Council of Canada (PIN:123817), the Fonds de Recherche du Québec-Nature et Technologies (FRQNT), the Canada Foundation for Innovation (CFI), and a Canada Research Chair. This research was undertaken thanks in part to funding from the Canada First Research Excellence Fund and the Gordon and Betty Moore Foundation's EPiQS Initiative (Grant No. GBMF5306 to L.T.). The National High Magnetic Field Laboratory is supported by the National Science Foundation through Grant No. NSF/DMR-1644779 and the State of Florida. J.-S.Z. was supported by NSF MRSEC under Cooperative Agreement No. DMR-1720595.
[1] B. Keimer, S. A. Kivelson, M. R. Norman, S. Uchida, and J. Zaanen, From quantum matter to high-temperature superconductivity in copper oxides, Nature 518, 179 (2015).

[2] C. Proust and L. Taillefer, The remarkable underlying ground states of cuprate superconductors, Annu. Rev. Condens. Matter Phys. 10, 409 (2019).

[3] S. Badoux et al., Change of carrier density at the pseudogap critical point of a cuprate superconductor, Nature (London) 531, 210 (2016).

[4] C. Collignon et al., Fermi-surface transformation across the pseudogap critical point of the cuprate superconductor $\mathrm{La}_{1.6-x} \mathrm{Nd}_{0.4} \mathrm{Sr}_{x} \mathrm{CuO}_{4}$, Phys. Rev. B 95, 224517 (2017).

[5] F. Laliberté et al., Origin of the metal-to-insulator crossover in cuprate superconductors, arXiv:1606.04491.
[6] B. Michon et al., Wiedemann-Franz law and Abrupt Change in Conductivity Across the Pseudogap Critical Point of a Cuprate Superconductor, Phy. Rev. X 8, 041010 (2018).

[7] M. Lizaire et al., Transport signatures of the pseudogap critical point in the cuprate superconductor $\mathrm{Bi}_{2} \mathrm{Sr}_{2-x} \mathrm{La}_{x} \mathrm{CuO}_{6+\delta}$, arXiv:2008.13692.

[8] C. Putzke et al., Reduced Hall carrier density in the overdoped strange metal regime of cuprate superconductors, Nat. Phys. (2021), doi: 10.1038/s41567-021-01197-0.

[9] B. Michon et al., Thermodynamic signatures of quantum criticality in cuprate superconductors, Nature (London) 567, 218 (2019).

[10] R. Daou et al., Linear temperature dependence of resistivity and change in the Fermi surface at the pseudogap 
critical point of a high- $T_{c}$ superconductor, Nat. Phys. 5, 31 (2009).

[11] R. A. Cooper et al., Anomalous criticality in the electrical resistivity of $\mathrm{La}_{2-x} \mathrm{Sr}_{x} \mathrm{CuO}_{4}$, Science 323, 603 (2009).

[12] A. Legros et al., Universal $T$-linear resistivity and Planckian dissipation in overdoped cuprates, Nat. Phys. 15, 142 (2019).

[13] M. Frachet et al., Hidden magnetism at the pseudogap critical point of a cuprate superconductor, Nat. Phys. 16, 1064 (2020).

[14] N. Doiron-Leyraud et al., Pseudogap phase of cuprate superconductors confined by Fermi surface topology, Nat. Commun. 8, 2044 (2017).

[15] C. E. Matt et al., Electron scattering, charge order, and pseudogap physics in $\mathrm{La}_{1.6-x} \mathrm{Nd}_{0.4} \mathrm{Sr}_{x} \mathrm{CuO}_{4}$ : An angle-resolved photoemission spectroscopy study, Phys. Rev. B 92, 134524 (2015).

[16] T. Yoshida et al., Universal Versus Material-Dependent TwoGap Behaviors of the High- $T_{c}$ Cuprate Superconductors: Angle-Resolved Photoemission Study of $\mathrm{La}_{2-x} \mathrm{Sr}_{x} \mathrm{CuO}_{4}$, Phys. Rev. Lett. 103, 037004 (2009).

[17] J. Chang et al., Anisotropic quasiparticle scattering rates in slightly underdoped to optimally doped high-temperature $\mathrm{La}_{2-x} \mathrm{Sr}_{x} \mathrm{CuO}_{4}$ superconductors, Phys. Rev. B 78, 205103 (2008).

[18] T. Kondo, T. Takeuchi, T. Yokoya, S. Tsuda, S. Shin, and U. Mizutani, Hole-concentration dependence of band structure in $(\mathrm{Bi}, \mathrm{Pb})_{2}(\mathrm{Sr}, \mathrm{La})_{2} \mathrm{CuO}_{6+\delta}$ determined by the angle-resolved photoemission spectroscopy, J. Electron Spectrosc. Relat. Phenom. 137-140, 663 (2004).

[19] S. Kawasaki, C. Lin, P. L. Kuhns, A. P. Reyes, and G.-q. Zheng, Carrier-Concentration Dependence of the Pseudogap Ground State of Superconducting $\mathrm{Bi}_{2} \mathrm{Sr}_{2-x} \mathrm{La}_{x} \mathrm{CuO}_{6+d}$ Revealed by ${ }^{63,65} \mathrm{Cu}$-Nuclear Magnetic Resonance in Very High Magnetic Fields, Phys. Rev. Lett. 105, 137002 (2010).

[20] W. Wu, M. S. Scheurer, S. Chatterjee, S. Sachdev, A. Georges, and M. Ferrero, Pseudogap and Fermi-Surface Topology in the Two-Dimensional Hubbard Model, Phys. Rev. X 8, 021048 (2018).

[21] H. Bragança, S. Sakai, M. C. O. Aguiar, and M. Civelli, Correlation-Driven Lifshitz Transition at the Emergence of the Pseudogap Phase in the Two-Dimensional Hubbard Model, Phys. Rev. Lett. 120, 067002 (2018).

[22] C. Collignon et al., Thermopower across the phase diagram of the cuprate $\mathrm{La}_{1.6-x} \mathrm{Nd}_{0.4} \mathrm{Sr}_{x} \mathrm{CuO}_{4}$ : Signatures of the pseudogap and charge-density-wave phases, Phys. Rev. B 103, 155102 (2021).

[23] K. Behnia, D. Jaccard, and J. Flouquet, On the thermoelectricity of correlated electrons in the zero-temperature limit, J. Phys.: Condens. Matter 16, 5187 (2004).

[24] K. Miyake and H. Kohno, Theory of quasi-universal ratio of seebeck coefficient to specific heat in zero-temperature limit in correlated metals, J. Phys. Soc. Jpn. 74, 254 (2005).

[25] F. Laliberté et al., Fermi-surface reconstruction by stripe order in cuprate superconductors, Nat. Commun. 2, 432 (2011).

[26] N. Doiron-Leyraud et al., Evidence for a small hole pocket in the Fermi surface of underdoped $\mathrm{YBa}_{2} \mathrm{Cu}_{3} \mathrm{O}_{y}$, Nat. Commun. 6, 6034 (2015).

[27] N. Doiron-Leyraud, C. Proust, D. LeBoeuf, J. Levallois, J.-B. Bonnemaison, R. Liang, D. A. Bonn, W. N. Hardy, and L. Taillefer, Quantum oscillations and the Fermi surface in an underdoped high- $T_{c}$ superconductor, Nature (London) 447, 565 (2007).

[28] O. Cyr-Choinière et al., Pseudogap temperature $T^{*}$ of cuprate superconductors from the Nernst effect, Phys. Rev. B 97, 064502 (2018).

[29] N. Doiron-Leyraud et al., Hall, Seebeck, and Nernst Coefficients of Underdoped $\mathrm{HgBa}_{2} \mathrm{CuO}_{4+\delta}$ : Fermi-Surface Reconstruction in an Archetypal Cuprate Superconductor, Phys. Rev. X 3, 021019 (2013).

[30] M. v. Zimmermann et al., Hard-x-ray diffraction study of charge stripe order in $\mathrm{La}_{1.48} \mathrm{Nd}_{0.4} \mathrm{Sr}_{0.12} \mathrm{CuO}_{4}$, Europhys. Lett. 41, 629 (1998).

[31] T. Niemöller, N. Ichikawa, T. Frello, H. Hünnefeld, N. H. Andersen, S. Uchida, J. R. Schneider, and J. M. Tranquada, Charge stripes seen with x-rays in $\mathrm{La}_{1.45} \mathrm{Nd}_{0.4} \mathrm{Sr}_{0.15} \mathrm{CuO}_{4}$, Eur. Phys. J. B 12, 509 (1999).

[32] N. K. Gupta et al., Vanishing nematic order beyond the pseudogap phase in overdoped cuprate superconductors, arXiv:2012.08450 (2020).

[33] J. Fink, V. Soltwisch, J. Geck, E. Schierle, E. Weschke, and B. Büchner, Phase diagram of charge order in $\mathrm{La}_{1.8-x} \mathrm{Eu}_{0.2} \mathrm{Sr}_{x} \mathrm{CuO}_{4}$ from resonant soft x-ray diffraction, Phys. Rev. B 83, 092503 (2011).

[34] O. Cyr-Choinière, D. LeBoeuf, S. Badoux, S. DufourBeauséjour, D. A. Bonn, W. N. Hardy, R. Liang, D. Graf, N. Doiron-Leyraud, and L. Taillefer, Sensitivity of $T_{c}$ to pressure and magnetic field in the cuprate superconductor $\mathrm{YBa}_{2} \mathrm{Cu}_{3} \mathrm{O}_{y}$ : Evidence of charge-order suppression by pressure, Phys. Rev. B 98, 064513 (2018).

[35] S. M. Souliou, H. Gretarsson, G. Garbarino, A. Bosak, J. Porras, T. Loew, B. Keimer, and M. Le Tacon, Rapid suppression of the charge density wave in $\mathrm{YBa}_{2} \mathrm{Cu}_{3} \mathrm{O}_{6.6}$ under hydrostatic pressure, Phys. Rev. B 97, 020503(R) (2018).

[36] I. Vinograd, R. Zhou, H. Mayaffre, S. Krämer, R. Liang, W. N. Hardy, D. A. Bonn, and M.-H. Julien, Nuclear magnetic resonance study of charge density waves under hydrostatic pressure in $\mathrm{YBa}_{2} \mathrm{Cu}_{3} \mathrm{O}_{y}$, Phys. Rev. B 100, 094502 (2019).

[37] Y. Zhu, Heat-loss modified Angstrom method for simultaneous measurements of thermal diffusivity and conductivity of graphite sheets: The origins of heat loss in Angstrom method, Int. J. Heat Mass Transf. 92, 784 (2016).

[38] H. Wang, F. Yang, Y. Guo, K. Peng, D. Wang, W. Chu, and S. Zheng, Determination of the thermopower of microscale samples with an ac method, Measurement 131, 204 (2019).

[39] M. Horio et al., Three-Dimensional Fermi Surface of Overdoped La-Based Cuprates, Phys. Rev. Lett. 121, 077004 (2018).

[40] Y. Fang et al., Fermi surface transformation at the pseudogap critical point of a cuprate superconductor, arXiv:2004.01725.

[41] J. a. N. Bruin, H. Sakai, R. S. Perry, and A. P. Mackenzie, Similarity of scattering rates in metals showing $T$-linear resistivity, Science 339, 804 (2013).

[42] H. v. Löhneysen, A. Rosch, M. Vojta, and P. Wölfle, Fermiliquid instabilities at magnetic quantum phase transitions, Rev. Mod. Phys. 79, 1015 (2007).

[43] R. Daou, O. Cyr-Choinière, F. Laliberté, D. LeBoeuf, N. Doiron-Leyraud, J.-Q. Yan, J.-S. Zhou, J. B. Goodenough, and L. Taillefer, Thermopower across the stripe critical point of $\mathrm{La}_{1.6-x} \mathrm{Nd}_{0.4} \mathrm{Sr}_{x} \mathrm{CuO}_{4}$ : Evidence for a quantum critical point in a hole-doped high- $T_{c}$ superconductor, Phys. Rev. B 79, 180505(R) (2009). 
[44] Y. Ando, Y. Kurita, S. Komiya, S. Ono, and K. Segawa, Evolution of the Hall Coefficient and the Peculiar Electronic Structure of the Cuprate Superconductors, Phys. Rev. Lett. 92, 197001 (2004).

[45] I. Tsukada and S. Ono, Negative Hall coefficients of heavily overdoped $\mathrm{La}_{2-x} \mathrm{Sr}_{x} \mathrm{CuO}_{4}$, Phys. Rev. B 74, 134508 (2006).

[46] H.-H. Klauss, W. Wagener, M. Hillberg, W. Kopmann, H. Walf, F. J. Litterst, M. Hücker, and B. Büchner, From Antiferromagnetic Order to Static Magnetic Stripes: The Phase Diagram of (La, $\mathrm{Eu})_{2-x} \mathrm{Sr}_{x} \mathrm{CuO}_{4}$, Phys. Rev. Lett. 85, 4590 (2000).

[47] S. Badoux et al., Critical Doping for the Onset of Fermi-Surface Reconstruction by Charge-Density-Wave Order in the Cuprate Superconductor $\mathrm{La}_{2-x} \mathrm{Sr}_{x} \mathrm{CuO}_{4}$, Phys. Rev. X 6, 021004 (2016).

[48] Q. Li, M. Hücker, G. D. Gu, A. M. Tsvelik, and J. M. Tranquada, Two-Dimensional Superconducting Fluctuations in
Stripe-Ordered $\mathrm{La}_{1.875} \mathrm{Ba}_{0.125} \mathrm{CuO}_{4}$, Phys. Rev. Lett. 99, 067001 (2007).

[49] Y. Nakamura and S. Uchida, Anisotropic transport properties of single-crystal $\mathrm{La}_{2-x-y} \mathrm{Nd}_{y} \mathrm{Sr}_{x} \mathrm{CuO}_{4}$ : Effect of the structural phase transition, Phys. Rev. B 46, 5841 (1992).

[50] M. Hücker, V. Kataev, J. Pommer, O. Baberski, W. Schlabitz, and B. Buchner, Consequences of stripe order for the transport properties of rare earth doped $\mathrm{La}_{2-x} \mathrm{Sr}_{x} \mathrm{CuO}_{4}$, J. Phys. Chem. Solids 59, 1821 (1998).

[51] J. Chang et al., Nernst and Seebeck Coefficients of the Cuprate Superconductor $\mathrm{YBa}_{2} \mathrm{Cu}_{3} \mathrm{O}_{6.67}$ : A Study of Fermi Surface Reconstruction, Phys. Rev. Lett. 104, 057005 (2010).

[52] M. K. Crawford, R. L. Harlow, S. Deemyad, V. Tissen, J. S. Schilling, E. M. McCarron, S. W. Tozer, D. E. Cox, N. Ichikawa, S. Uchida, and Q. Huang, High-pressure study of structural phase transitions and superconductivity in $\mathrm{La}_{1.48} \mathrm{Nd}_{0.4} \mathrm{Sr}_{0.12} \mathrm{CuO}_{4}$, Phys. Rev. B 71, 104513 (2005). 